\title{
Medline at thirty-five
}

\author{
Derek Richards \\ Editor, Evidence-based Dentistry
}

Evidence-Based Dentistry (2006) 7, 59. doi: 10.1038/sj.ebd.6400440

The Medline database is one of the key resources that has enabled the development of evidence-based practice. The availability of Medline and other electronic databases, Embase, LILACS, CINHAL and the Cochrane Library, to name but a few of the better known ones, has improved no end the accessibility of the accumulated wisdom in the biomedical literature.

When Medline was launched on 27 October 1971 it featured 239 indexed journals and had a mere 25 users. At that stage, although you could usually obtain what you were looking for, it required determination to master its intricacies and deal with its idiosyncrasies. This improved in the 1980s, with the introduction of the software programme Grateful Med, enabling searching from home at an average of $\$ 2$ per search. Many considered this a bargain because trying to find the same information in the printed Index Medicus (which preceded Medline) would surely cost much more in time and effort, if it could be done at all.

The 1990s saw the introduction of the PubMed interface, which had its 10th anniversary in January 2006, with the introduction of free Medline searching via the Web by US Vice-President Gore. Free access and the simplified searching interface of PubMed have allowed an enormous increase in the number of searches being carried out on Medline. Free provision of this enormous resource is arguably the US's greatest contribution to modern healthcare. It is expected that during 2006, Medline/ PubMed will average 77 million unique visits and about 800 million searches of the 4928 journals now indexed. Of these searches it is estimated that 30 per cent are being conducted by consumers rather than healthcare professionals. This led to the development of MedlinePlus (to be found at medlineplus.gov), a service for consumers offering selected Medline results and other health information.

Although Medline is a key resource for the committed evidence-based practitioner, it is just one player in the resources available. Haynes $^{1}$ describes these as a " $4 S$ " hierarchical system of studies, syntheses, synopses and systems. Studies form the base, ie, syntheses (systematic reviews); at the next level are synopses of studies; synthesis is above them; and evidence-based systems are found at the top. Evidence-based practitioners should look for information at the highest level to help them address their clinical problems. The aim of this journal is to provide synopses for practitioners.

Whereas medicine has seen the development of some systems, such as Clinical Evidence (www.clinicalevidence.org) and UpToDate (www.uptodate.com), that are effective electronic textbooks which integrate evidence-based information about specific clinical problems and undergo regular updating, similar systems in dentistry have yet to develop. Clinical Evidence does contain some information on a few dental topics (aphthous ulcers, burning mouth syndrome, oropharyngeal candidiasis, halitosis, impacted wisdom teeth and herpes labialis) and the National Library for Health in the UK has a range of specialist libraries including one for Oral Health (www.library.nhs. uk/oralhealth/).

The top of Haynes's hierarchy is the evidence-based clinical information system that would integrate and summarise concisely all the relevant and important research evidence related to a clinical problem and would automatically link, through an electronic medical record, a specific patient's circumstances to the relevant information. The role of such a system is not to tell clinicians what to do, because they would need to integrate the system's evidence with the patient's circumstances and wishes ${ }^{2}$ to be practicing in an evidence-based manner.

We have had computers in dental practices for many years now and they have tended to be used most for administrative functions. Clinical management systems are now becoming increasingly widespread. Some dental tools are available, for example: Evidents (medinformatics.uthscsa.edu/EviDents/), a 'PICO' (Problem, Intervention, Comparision, Outcome)-based ${ }^{3}$ search engine for Medline; and Evidentista (www.evidentista.org/), which is a site that provides clinical bottomline answers to a range of clinical problems and which allows the user to access further details as required. These types of development point the way to the type of systems Haynes was describing, and to the possibility of a more evidence-based future.

1. Haynes RB. Of studies, syntheses, synopses, and

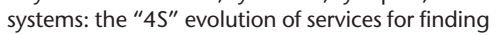
current best evidence. Am Coll Physician J Club 2001; 134 :A11-A13.

2. Haynes RB, Sackett DL, Gray JR, Cook DL, Guyatt $\mathrm{GH}$. Transferring evidence from research into practice. 1 . The role of clinical care research evidence in clinical decisions [editorial]. Am Coll Physicians J Club. 1996; 125 :A14-A6; Evid based Med 1996; 1:196-198.

3. Richardson WS, Wilson MC, Nishikawa J, Hayward RS. The well-built clinical question: a key to evidence-based decisions. Am Coll Physicians J Club 1995; 123 :A12-A13. 